\title{
Note on Mental Division by Large Numbers.
}

By J. Taylor, M.A.

Since $\frac{\mathbf{A}}{\mathbf{B}}=\frac{n \mathbf{A}}{n \mathbf{B}}$, it is possible to divide mentally by many numbers, integral or fractional.

$$
\begin{array}{ll}
\text { Examples : } & \frac{3275}{125}=\frac{8.3275}{8.125}=\frac{26200}{1000}=26 \cdot 2 ; \\
\frac{4579}{14^{2}}=\frac{7.4579}{7.14 \frac{2}{7}}=\frac{32053}{100}=320 \cdot 53 .
\end{array}
$$

In 1901, when I was drawing up notes on Mental Arithmetic, I looked into many text-books in search of a simple method for dividing by such numbers as $19,29,99,87$, etc., but found none. The following method, viz., that of using a multiple of ten as dirisor instead of a given divisor, was then discovered by me, and I think it simple enough to be learned and practised by any one.

If it be required to divide $A$ by $D$, let the quotient at any step be $Q$, then the product at that step will be $D Q$, and the remainder, $\mathbf{R}=\mathbf{A}-\mathbf{D Q}$, where $\mathbf{R}$ cannot exceed $\mathbf{D}$ nor be less than zero.

Instead of working with $\mathrm{D}$ as divisor, take as divisor $d$, that multiple of ten which is nearest to $D$, and if, at each step, care be taken with $Q$, so that $R$ never exceeds $D$ nor is less than zero, the required quotient $Q$ will be obtained.

Dividing by $d$, the remainder at any step is $r=\mathrm{A}-d \mathrm{Q}$,

$$
\text { and since } \quad \mathrm{R}=\mathrm{A}-\mathrm{DQ} \text {, and } r=\mathrm{A}-d \mathbf{Q}_{\mathrm{i}}
$$

$$
\mathbf{R}=r+(d-\mathbf{D}) \mathbf{Q} \text {. }
$$

Thus $R$, the remainder which would have been obtained on dividing by $\mathrm{D}$, is obtained at every step by adding $(d-\mathrm{D}) Q$ to $r$, the remainder obtained on dividing by $d$. The importance of obtaining $\mathbf{R}$ correctly at each step is so great that $I$ would suggest that the method of obtaining it in each sum be made the key-word of that sum.

For example: In dividing by $19,29,39,49$, or 99 , the divisor used is $20,30,40,50$, or 100 , where $d-\mathrm{D}=1$, so that $\mathrm{R}=r+Q$, and the key-word in such examples would be : Add once $Q$. 
In dividing by 67,87 or 97 , the divisor used is 70,90 or 100 , where $d-\mathrm{D}=3$, and $\mathrm{R}=r+3 \mathrm{Q}$, and the key-word would be: $A d d$ three times $Q$.

Again, in dividing by 31,61 or 71 the divisor used would be 30,60 or 70 , where $d-\mathrm{D}=-1$, and $\mathrm{R}=r-\mathrm{Q}$, and the key-word would be: Subtract once $Q$.

In $43,53,73$ or 83 the key-word would be : Subtract three times $Q$.

The divisors used in working the following examples are printed in heavier type.

\begin{tabular}{|c|c|c|c|c|c|}
\hline \multirow{2}{*}{\multicolumn{6}{|c|}{$2019 \frac{451360}{23755^{15}}$}} \\
\hline & & & & & \\
\hline \multicolumn{2}{|c|}{ Divide by 20} & Quot. & \multicolumn{3}{|c|}{$r+Q=R$} \\
\hline & 45 & 2 & \multicolumn{2}{|c|}{$\overline{5}+2$} & 7 \\
\hline & 71 & 3 & \multicolumn{2}{|c|}{$11+3$} & 14 \\
\hline & 143 & 7 & \multicolumn{2}{|c|}{$3+7$} & 10 \\
\hline & 106 & $\overline{5}$ & \multicolumn{2}{|c|}{$6+5$} & 11 \\
\hline & 110 & 5 & \multicolumn{2}{|c|}{$10+5$} & 15 \\
\hline 100 & \multicolumn{2}{|c|}{$99 \frac{\mid 223134}{2253^{*}}$} & 80 & \multicolumn{2}{|c|}{$79 \frac{\mid 763794}{9668 \frac{29}{7}}$} \\
\hline 1000 & \multicolumn{2}{|c|}{$999 \mid \frac{23+1527}{2343}$} & 120 & 119 & $\frac{2785857}{23410}$ \\
\hline
\end{tabular}

$7067 \frac{4462182}{66599 \frac{1}{67}}$ Key-word: Add three times $Q$.

$\begin{array}{rccr}\text { Divide by } 70 & \text { Quot. } & r+3 Q=\mathrm{R} \\ 446 & 6 & 26+18 & 44 \\ 442 & 6 & 22+18 & 40 \\ 401 & 5 & 51+15 & 66 \\ 668 & 9 & 38+27 & 65 \\ 652 & 9 & 22+27 & 49\end{array}$
$60 \quad 57 \frac{j 12623}{548435}$
$100 \quad 37 \frac{\mid 236582}{2438 \frac{96}{7}}$
$90 \quad 87 \frac{5628435}{64694 \frac{57}{87}}$
$400397 \mid 2847928$

$10 \mathrm{Vol}, 21$ 
Key-word: Subtract once ?, $R=r-Q$.

$$
1011 \frac{37636876}{3421534 \frac{2}{1 T}} \quad 3031 \frac{285670357}{9215172 \frac{25}{31}}
$$

Key-word: Subtract three times $Q ; \mathrm{R}=r-3 \mathrm{Q}$.

$$
7073 \frac{\mid 6249683}{85612 \frac{7}{63}} \quad 5053 \frac{\mid 1286494}{24273 \frac{25}{53}} .
$$

In the following examples, the figures in large type show where special care has to be taken with $Q$, so that the remainder $R$ may neither exceed the divisor $\mathrm{D}$ nor be less than zero.

$$
\begin{aligned}
& 1011 \frac{\mid 478265}{43478 ?}
\end{aligned}
$$

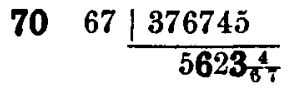

$$
\begin{aligned}
& 10 \quad 9 \frac{32876}{3652 \frac{8}{9}} \\
& 7068 \frac{\mid 156675}{2304 \frac{3}{68}} \\
& \frac{11}{11}=\cdot \dot{2} 682 \dot{9} \\
& \frac{\overline{7}}{17}=\cdot \dot{2} 9411764 \\
& 4043 \frac{\mid 23572864}{548206 \frac{6}{45}} \\
& 30 \quad 29 \frac{149386}{5161^{7}} \\
& 10096 \frac{327654}{3413_{96}^{6}} \\
& 1000998 \frac{568976}{570 \frac{11 \frac{1}{19}}{8}} \\
& 40 \quad 41 \frac{\mid 11 .}{\cdot 26829} \\
& 20 \quad 17 \frac{5^{5}}{29411764} \\
& 45243 \text { farthings }=£ 47 " 2 " 63 \\
& 1000960 \frac{\mid 45243}{£ 45}+2043 \text { farthings } \\
& =£ 45+£ 2+123 \text { farthings } \\
& =£ 47 " 2 \text { " } 63 \\
& 367234 \text { farthings }=£ 382 " 10 " 8 \frac{1}{2} \\
& 1000960 \frac{367234}{£ 382}+514 \text { farthings } \\
& 46236 \mathrm{lb} .=412 \mathrm{cwt} .92 \mathrm{lb} \text {. } \\
& 3724562 \mathrm{lb} .=1662 \text { tons } 15 \mathrm{cwt} \text {. \& lb. } \\
& 110112 \frac{46236}{412} \text { cwt. } 92 \text { lb. } 110112 \frac{3724562}{33255} \text { cwt. } 2 \text { lb. }
\end{aligned}
$$


It is evident that suws in Long Division may be much simplitied by the adoption of this method. The remainder $R=r+(d-D) Q$ nay be obtained mentally

$$
\begin{aligned}
& \left.\begin{array}{l}
397 \\
400
\end{array}\right) \begin{array}{l}
284792857 \\
2800
\end{array} \\
& 689 \\
& 400 \\
& 2922 \\
& 2800 \\
& 1438 \\
& \frac{1200}{2475} \quad \frac{4000}{2827} \\
& \frac{2400}{937} \quad \frac{2500}{4329} \\
& \frac{800}{143} \quad \frac{4500}{18}
\end{aligned}
$$

